\title{
COMPLICAÇÕES BUCAIS DA RADIOTERAPIA NO TRATAMENTO DO CÂNCER DE CABEÇA E PESCOÇO
}

\author{
COMPLICATIONS OF RADIOTHERAPY IN THE TREATMENT OF HEAD \\ AND NECK CANCER
}

\section{Vanessa Divina Pires De Oliveira}

Discente do Curso Superior de Tecnologia em Radiologia, Faculdade Evangélica de Ceres, Ceres-Goiás.

Email: vanessapires_ng@hotmail.com

\section{Danielle Muniz Pessoa Aires}

Especialista em Vigilância Sanitária e Controle de Qualidade de Alimentos.

Docente do Curso Superior de Tecnologia em Radiologia, Faculdade Evangélica de Ceres, Ceres-Goiás.

Email: daniellempessoa@ hotmail.com

\section{RESUMO}

INTRODUÇÃO: O câncer de cabeça e pescoço compreende as neoplasias que ocorrem na cavidade bucal e nasal, face, faringe, laringe e glândulas. A irradiação na região de cabeça e pescoço acarreta danos aos tecidos sadios, resultando em manifestações bucais, como candidose bucal, disgeusia, cáries por radiação, mucosite, xerostomia, trismo e osteorradionecrose. OBJETIVOS: Discorrer sobre as complicações bucais decorrentes da radioterapia na região de cabeça e pescoço, descrevendo os principais fatores de risco e efeitos colaterais do tratamento, assim como enfatizar a importância do odontólogo durante o tratamento radioterápico. METODOLOGIA: Trata-se de um trabalho descritivo e de aspecto qualitativo de revisão de literatura, por meio de revistas, artigos científicos e livros. Utilizouse principalmente busca ativa nas bases de dados virtuais Scielo e Google Acadêmico. RESULTADOS E DISCUSSÃO: A radioterapia é uma forma de tratamento utilizada para o tratamento dos tumores malignos de cabeça e pescoço, utilizando feixe de radiações ionizantes. São muitos os efeitos colaterais causados no tratamento radioterápico podendo apresentar irritação, feridas na boca e vermelhidão, boca seca ou saliva espessa, dificuldade para engolir, alterações no paladar ou náuseas, fadiga e diarreia. Os pacientes que são submetidos a esse tratamento, desenvolvem complicações bucais, sendo necessário o acompanhamento do odontólogo em todas as fases do tratamento. CONCLUSÃO: Manifestações bucais e efeitos colaterais merecem atenção especial, pois comumente ocorrem em decorrência do tratamento radioterápico do câncer de cabeça e pescoço. Estudos neste sentido são importantes tanto para definir a conduta terapêutica, quanto para alívio ou diminuição do desconforto causados aos pacientes. 
Palavras-Chave: Câncer de cabeça e pescoço; Complicações bucais; Efeitos colaterais; Radioterapia.

\begin{abstract}
INTRODUCTION: Head and neck cancer comprises neoplasms that occur in the buccal and nasal cavity, face, pharynx, larynx and glands. Irradiation in the head and neck region causes damage to healthy tissues, resulting in oral manifestations such as oral candidosis, dysgeusia, radiation caries, mucositis, xerostomia, trismus and osteorradionecrosis. OBJECTIVES: To describe the oral complications resulting from radiotherapy in the head and neck region, describing the main risk factors and side effects of the treatment, as well as emphasizing the importance of the dentist during radiotherapy treatment. METHODOLOGY: This is a descriptive and qualitative review of literature, through magazines, scientific articles and books. We used mainly active search in the virtual databases Scielo and Google Scholar. RESULTS AND DISCUSSION: Radiotherapy is a form of treatment used for the treatment of malignant tumors of the head and neck using a beam of ionizing radiation. There are many side effects caused by radiation therapy, which may include irritation, mouth sores and redness, dry mouth or thick saliva, difficulty swallowing, changes in taste or nausea, fatigue and diarrhea. Patients who undergo this treatment develop oral complications and it is necessary to follow the dentist at all stages of treatment. CONCLUSION: Oral manifestations and side effects deserve special attention, as they commonly occur as a result of the radiotherapy treatment of head and neck cancer. Studies in this sense are important both to define the therapeutic course, and to relieve or reduce the discomfort caused to patients.
\end{abstract}

Key words: Head and neck cancer; Oral complications; Radiotherapy.

\title{
INTRODUÇÃO
}

Câncer é a denominação dada a um grupo de mais de 100 doenças, tendo em comum à multiplicação e o crescimento desordenado das células que envolvem tecidos e órgãos, e podem se espalhar para outras partes do corpo. Dessa forma, as células se dividem rapidamente, com tendência a serem muito agressivas e incontroláveis, levando ao desenvolvimento de neoplasias ou tumores malignos (INCA, 2011). Muitos fatores estão incluídos no desenvolvimento do câncer de cabeça e pescoço, sendo que o tabagismo e alcoolismo são os fatores de risco mais significativos (BRAGANTE et al., 2011).

O câncer de cabeça e pescoço compreende as neoplasias que ocorre nos sítios anatômicos da cavidade bucal, face, cavidade nasal, faringe, glândulas e laringe (COLOMBO; RAHAL, 2009). A radioterapia, juntamente com a quimioterapia e a cirurgia, são as principais modalidades de tratamento. Essa modalidade de tratamento é usada em mais da metade de todos os tratamentos contra o câncer e, em alguns casos, é o tratamento preferido e mais eficaz de todos (SILVA et al., 2011). 
A radioterapia é uma forma de tratamento utilizada para o tratamento dos tumores malignos da cabeça e pescoço. Dessa forma, as doses altas de radiação em amplos campos que incluem a cavidade oral, mandíbula, maxila, e glândulas salivares constantemente ocasionam em diversos efeitos indesejados (JHAM; FREIRE, 2006). O tratamento pode ser realizado em duas modalidades: a braquiterapia, que utiliza isótopos radioativos em contato direto com o tumor, e a teleterapia, onde utiliza uma fonte externa colocada à distância do paciente. A escolha de modalidade depende do tipo de câncer e de sua profundidade (SALAZAR et al., 2008).

Segundo Pelisser et al. (2008), a irradiação na região de cabeça e pescoço acarreta danos aos tecidos sadios, que podem resultar em sequelas bucais, como candidose bucal, disgeusia, cáries por radiação, mucosite, xerostomia, trismo e osteorradionecrose, que ainda podem ser divididos em agudos e tardios, reversíveis e irreversíveis. Etilismo e tabagismo ainda são os fatores principais de risco, existem outros fatores podem induzir o desenvolvimento do carcinoma de cabeça e pescoço. Assim o profissional de odontologia tem um papel importante no atendimento dos pacientes oncológicos, sendo indispensável sua participação do planejamento das diferentes fases de tratamento dessas lesões, sendo essencial sua participação na melhoria de qualidade de vida desses pacientes (FREITAS et al., 2011).

Para Arisawa et al. (2005) as complicações orais do câncer se encontram entre as mais devastadoras a curto e longo prazo, por afetarem as atividades humanas mais básicas, tais como alimentar-se e comunicar-se, portanto, não surpreende que os pacientes com estes problemas possam se isolar do contato interpessoal e das relações sociais, e apresentarem depressão clínica como resultado destas dificuldades e frustrações que encontram no seu diaa-dia. Para diversos pacientes, é um tratamento bastante eficaz, podendo fazer com que o tumor seja eliminado e a doença controlada, ou até mesmo curada. Quando não é possível alcançar a cura, a radioterapia pode colaborar melhorando a qualidade de vida. Isso acontece porque as aplicações minimizam o tamanho do tumor, o que alivia a pressão, dores e outros sintomas, possibilitando um alívio e conforto aos pacientes (KARKOW et al., 2013).

O objetivo deste estudo foi discorrer sobre complicações bucais decorrentes da radioterapia na região de cabeça e pescoço, descrevendo os principais fatores de risco e efeitos colaterais do tratamento, assim como enfatizar a importância do odontólogo durante o tratamento.

\section{METODOLOGIA}

REFACER v 7. , n. 1, 2018. ISSN - 2317-1367 
Trata-se de um trabalho descritivo e de aspecto qualitativo de revisão de literatura, por meio de revistas, artigos científicos e livros. Utilizou-se principalmente busca ativa nas bases de dados virtuais Scielo e Google Acadêmico.

Foram utilizados 31 artigos, dos quais 18 foram descartados, tendo como critérios de exclusão: artigos muito antigos e não abrangendo o tema com clareza. Assim, foram citados artigos contendo: definições de alguns efeitos e reações adversas durante e após o tratamento radioterápico, modalidades de tratamento, os fatores de risco, a importância do odontólogo e possíveis complicações da radioterapia e as suas influências na qualidade de vida dos pacientes.

\section{REVISÃO BIBLIOGRÁFICA}

Diferentes tipos de tumores são capazes de se manifestar na região de cabeça e pescoço, no entanto, o carcinoma de células escamosas ou carcinoma espinocelular correspondem de $90 \%$ a 95\% dos casos. Esse tipo de câncer em nível mundial equivale cerca de $10 \%$ dos tumores malignos, envolvendo muitos sítios, que numa média $40 \%$ dos casos surgem na cavidade bucal, $25 \%$ na laringe, $15 \%$ na faringe, $7 \%$ nas glândulas salivares e $13 \%$ nos outros locais (FREITAS et al., 2011; CASTRO, 2017).

Uma parte considerável dos cânceres de cabeça e pescoço começa nas células escamosas que envolvem as superfícies úmidas dessa região, como exemplo, dentro da boca, do nariz e da garganta. Os tumores de cabeça e pescoço também podem começar nas glândulas salivares, mas são bem raros. As glândulas salivares contêm muitos tipos de células que podem tornar-se cancerosas, de modo que existem diferentes tipos de cânceres de glândulas salivares. Os sintomas referentes dessas neoplasias incluem manifestação de nódulos, dor de garganta, complicação para engolir, alteração ou rouquidão na voz e feridas que não cicatrizam (ONCOGUIA, 2014).

Segundo o Instituto Nacional do Câncer (INCA, 2016), estimou-se que para o Brasil, no ano de 2016, 11.140 novos casos de câncer da cavidade oral em homens, e 4.350 em mulheres. Esses valores equivalem a um risco estimado de 11,27 novos casos a cada 100 mil homens e 4,21 a cada 100 mil mulheres. Além disso, o diagnóstico desses pacientes é realizado em estádios clínicos evoluídos da doença, o que normalmente se estabelece em uma elevada taxa de deformidades e sequelas, dessa forma, reduzindo a sua qualidade de vida. A 
junção desses fatores acaba tornando desagradável o tratamento e qualifica esse tipo de câncer como significativa questão de saúde pública (MARTA et al., 2011).

Os tratamentos para controle dessas neoplasias são a cirurgia de cabeça e pescoço, a quimioterapia e a radioterapia. Frequentemente, a cirurgia é o tratamento escolhido, podendo ou não ser associada à radioterapia, e a quimioterapia. No entanto, o tratamento instituído dependerá da localização, estadiamento clínico, tipo histológico da neoplasia e condições saúde do paciente e se o mesmo está disposto a contribuir com o tratamento, devendo informa-lo sobre os efeitos colaterais. No local que é irradiado o tumor, é conhecido como campo de radiação, e o ponto central receberá a dose estabelecida pelo radioterapeuta e os tecidos próximos irão receber doses menores de radiação (LÔBO; MARTINS, 2009; SASSI; MACHADO, 2009).

Isso ocorre em decorrência da falta de seletividade desse método terapêutico para com as células malignas, onde as células saudáveis são também afetadas (GRIMALDI et al., 2005). Durante a radioterapia são usados raios em alta energia para erradicar as células do câncer. É o tratamento primário para alguns tumores pequenos, podendo ser usada depois da cirurgia para ter certeza de que todas as células cancerígenas foram destruídas (VIDAL; REVOREDO, 2010).

Conforme Almeida et al. (2008), a radioterapia é considerada um método capaz de destruir células tumorais utilizando feixe de radiações ionizantes, na qual uma dose précalculada de radiação é empregada, num determinado tempo, a um volume do tecido que compreenda o tumor, buscando eliminar as células tumorais totalmente, causando se possível um dano menor nas células normais próximas. Quando essa radiação ocorre na região de cabeça e pescoço, produz mudanças reversíveis e irreversíveis dependendo da fonte, das doses e do campo de radiação, ocorrendo principalmente na mucosa, nas glândulas salivares, nas estruturas de suporte dental e nos dentes (SANTOS et al., 2010).

Paula e Sawada (2015) relataram que a radioterapia apresenta três objetivos principais, o curativo, o remissivo e o sintomático. A radioterapia quando é utilizada para extinguir todo o tumor, dizemos que é curativo, quando o objetivo é reduzir parte do tumor, ou é complementar ao tratamento quimioterápico e cirúrgico, dizemos que é remissivo e o objetivo da radiação sintomática, é de reduzir dores localizadas. Para muitos pacientes esse tratamento é muito eficiente, pode ser que o tumor desapareça e a doença fique controlada, ou até curada. Contudo, senão obter a cura, a radioterapia contribui na melhora da qualidade de 
vida, diminuindo o tamanho do tumor, aliviando a pressão, reduzindo hemorragias, dores e demais sintomas, proporcionando alívio e conforto aos pacientes (KARKOW et al., 2013).

A radioterapia constitui-se na utilização da radiação ionizante nas neoplasias malignas, consistindo em duas modalidades: braquiterapia e a teleterapia. A braquiterapia (brachys, do grego "em contato"), em que fontes de radiação são colocadas em contato direto ou a uma distância muito próxima do tecido com tumor, enquanto a teleterapia, (tele, do latim "a uma distância"), conhecida também como radioterapia externa é realizada com um aparelho emissor de radiação, que são os aceleradores lineares ou aparelhos de cobalto (MARTA et al., 2011).

$\mathrm{Na}$ braquiterapia, se utiliza um radioisótopo que emite raios gama, sendo colocado em contato direto com o tumor, sendo, intracavitário ou intersticial. A quantidade de radiação fornecida para destruir o tumor é limitada devido aos riscos de danos a tecidos sadios próximos. Para aumentar a dose de radiação fornecida, é necessário empregar fontes pequenas de radiação diretamente em contato com a área lesionada pelo tumor (GRIMALDI et al., 2005). A sua vantagem é que, nessa modalidade, pode-se irradiar o tumor com doses altas, mas protegendo os órgãos e estruturas que o envolvem. Essa forma terapêutica tem se desenvolvido nos últimos anos, utilizando sistemas de alta tecnologia, pretendendo-se diminuir o tempo de exposição, tornando o tratamento mais prático, rápido e ágil (FREITAS et al., 2011).

A teleterapia é o método em que a fonte de radiação é emitida pelo equipamento a uma distância que equivale de 80 a $100 \mathrm{~cm}$ da pele do paciente, depende da região a ser tratada, sendo direcionada ao tumor. Os aparelhos principais nessa modalidade são os aparelhos de telecobaltoterapia e os aceleradores lineares (FREITAS et al., 2011; MARTA et al., 2011). Antes de iniciar as aplicações da radioterapia externa, uma equipe multiprofissional faz um planejamento do tratamento que será realizado, definindo uma estratégia a ser seguida e o local a ser tratado. Posteriormente definem aspectos como dose e intervalo do tratamento, assim como data de início e horário das aplicações. O valor da dose total é dividido em aplicações diárias, cerca de cinco a seis semanas (FRIGATO; HOGA, 2003).

Marcas de tinta na pele do paciente são feitas durante o planejamento da teleterapia, para delimitar o local a ser tratado, e o mesmo é orientado a manter essas marcas até finalizar o tratamento, e, sendo possível que continue tomando banho como de costume, é preciso ter cuidado e não esfregar a pele na região demarcada. Se por acaso as marcas deixarem de ser nítidas, o técnico deverá reforçar essas marcas. No término das aplicações da 
terapia, as marcas podem ser retiradas cuidadosamente lavando o local, com a cautela de não causar lesões na pele (FRIGATO; HOGA, 2003).

\section{FATORES DE RISCO}

As complicações que ocorrem durante o tratamento radioterápico dependem, além da quantidade total da dose, de outros fatores como: radiossensibilidade e tipos dos tecidos saudáveis envolvidos pela radiação, idade, condições sistêmicas, do fracionamento da dose e, principalmente, de condições que podem compactuar com a integridade da mucosa oral, como uma prótese mal adaptada, alguma doença dentária existente e de uma inadequada higiene bucal (SALAZAR et al., 2008).

Galbiatti et al. (2013), afirmam que o tabagismo e o etilismo são os fatores principais no desenvolvimento do câncer de cabeça e pescoço. Esse risco é relacionado com a intensidade e de consumir bebidas alcoólicas e o hábito de fumar. Quando o cigarro é associado ao consumo de álcool aumenta em 40 vezes o risco dos carcinomas de cabeça e pescoço se manifestarem. O cigarro contém aproximadamente 4.700 substâncias, dessas 50 das quais carcinogênicas. Porém, nem todos os tabagistas e consumidores de álcool tendem a desenvolver câncer de cabeça e pescoço, o que indica que a variação individual da vulnerabilidade genética desempenha um papel crítico. Existe ainda uma forte ligação entre uso de fumo e álcool, considerando que o uso combinado eleva o risco ainda mais.

Para Bragante et al. (2011) vários são os fatores envolvidos para desenvolver-se os carcinomas de cabeça e pescoço, onde se inclui a predisposição genética, a exposição a fibras têxteis, níquel e couro, hábitos sociais e sexuais, infecções pelo papilomavírus humano (HPV), sendo que o consumo de bebidas alcoólicas e tabaco são os fatores de risco mais significativos. Aspectos como sexo masculino, idade acima de 40 anos, e raça branca também são considerados como fatores predisponentes ao seu desenvolvimento.

\section{EFEITOS COLATERAIS DA RADIOTERAPIA}

Os efeitos colaterais da radioterapia irão depender da idade, volume da área irradiada, dose total, quantidade de radiação administrada, local irradiado, fracionamento, das condições clínicas do paciente e dos tratamentos associados. A radiação ionizante tem capacidade de produzir efeitos nocivos na mucosa oral e demais proximidades, resultando em desconforto aos pacientes, dificultando ou restringindo as suas atividades normais e 
interferindo de maneira significativa na vida dos pacientes (RAGGHIANTI et al., 2002; BRAGANTE et al., 2011).

Os efeitos agudos ocorrem nas primeiras semanas após o inicio da radioterapia e prevalecem nos tecidos que mostram alto índice de proliferação celular. Os principais efeitos agudos que ocorrem no decorrer do tratamento da radioterapia são: mucosite, disfagia, disfonia, xerostomia, alteração do paladar, infecções e perda de peso (BRENTANI; COELHO; KOWALSKI, 2003; SAWADA et al., 2006; MARTA et al., 2011).

Os efeitos tardios acontecem de meses a anos após o término da irradiação. São tecidos que apresenta pouca atividade mitótica, de resposta prolongada, relacionada à morte clonogênica de células e menos vulnerabilidade a apoptose (MARTA et al., 2011). Efeitos tardios se destacam a ulceração da mucosa, cáries de radiação, trismo, fístulas, alterações hormonais, queda de cabelo, mielite cervical, osteorradionecrose, atrofia dos tecidos, alterações auditivas e oftalmológicas, edema, atenuação do fluxo de saliva e perda dos dentes. Em geral, quanto maior a dose por fração de radioterapia, maior a probabilidade de ocorrerem efeitos tardios (SAWADA et al., 2006; ROLIM et al., 2011).

Esses efeitos ocorrem durante o curso do tratamento, porém, apresentam uma atenuação com a utilização de medicação, e em casos mais extremos é necessária à interrupção do tratamento. As doses altas de radiação causam ainda hipóxia, necrose e vulnerabilidade para infecção. No local de irradiação da pele, podem apresentar como consequências, descamação, eritema, necrose, formação de bolhas, dor e ardência. A dor causada dificulta na alimentação podendo levar à perda de peso, caquexia, desidratação, anorexia (ROLIM et al., 2011).

\section{MANIFESTAÇÕES BUCAIS RELACIONADOS À RADIOTERAPIA}

\section{Xerostomia}

A xerostomia, que é a sensação de boca seca, pode ser provocada pela diminuição ou não da função das glândulas salivares, que devido à radiação, lesam as glândulas salivares, conduzindo eventualmente para fibrose e hipofunção secretora com alteração na quantidade e na qualidade da saliva (FREITAS et al., 2011). As salivas nos pacientes irradiados podem reduzir em até $90 \%$, tornando a saliva viscosa e espessa. Em casos mais ressaltados, os

pacientes retratam que a mucosa está sem nenhuma umidificação, o que acaba dificultando a 
mastigação e a preparação do bolo alimentar, e assim, a ingestão e até mesmo a fala (ROLIM et al., 2011).

Nessa complicação, o fluxo salivar encontra-se num nível menor que $0,3 \mathrm{ml} / \mathrm{min}$, causando alteração da gustação, perda do apetite e do peso, disfagia, influenciando de forma adversa à qualidade de vida do paciente, uma vez que não ocorre mais a liquefação e lubrificação dos alimentos, que associados à irritação da mucosa, tornam a deglutição dolorosa (SALAZAR et al., 2008; LÔBO; MARTINS, 2009). Esta situação pode estimular doenças bucais severas e deficiência nutricional causando um impacto significativo na qualidade de vida, não havendo dúvida que a xerostomia é a causa principal de enfermidades bucais nos pacientes (FREITAS et al., 2011).

\section{Osteorradionecrose}

Segundo Monteiro et al., (2005), a osteorradionecrose é causada por uma necrose óssea induzida pela radiação, onde o tecido ósseo tem a sua capacidade de alteração e cicatrização prejudicada em carácter definitivo. Os primeiros sinais ou sintomas surgem frequentemente, meses ou anos após a exposição à radiação. Caccelli e Rapoport (2008) relatam que, a osteoradionecrose é uma das manifestações bucais mais graves e sérias decorrentes da radioterapia. Sua ocorrência depende de vários aspectos, como qualidade, quantidade e fracionamento da dose total da radiação administrada, local do tumor a ser irradiada, a qualidade dental, periodontal, tecidos e grau do trauma entre os tecidos irradiados, principalmente a mucosa bucal.

Com incidência maior em idosos, num percentual de $10 \%$ a $37 \%$ dos casos, ocorre sete vezes mais na mandíbula do que na maxila, pela sua alta densidade óssea e menos vascularização, podendo ocorrer até dois anos após ter o fim da radioterapia (ROLIM et al., 2011). Suas características clínicas: ulceração da mucosa com exposição óssea e presença de dor, sendo a mandíbula mais acometida que a maxila. $\mathrm{O}$ diagnóstico se dá pela avaliação clínica e radiográfica devido à existência de áreas de neoformação e reabsorção óssea (FREITAS et al., 2011).

\section{Cáries de radiação}

As cáries surgem de 3 a 12 semanas após a radioterapia, causa predominante para essas lesões se desenvolverem é devido à redução da quantidade e qualidade da saliva, causando um efeito direto sobre os dentes que são alterados pela radiação, tornando-os mais 
propensas à descalcificação (SALAZAR et al., 2008). As lesões são defeitos que afetam superfícies geralmente resistentes à cárie, em regiões lisas. Pacientes com baixa atividade de cárie começar a apresentá-la em muitos níveis de destruição dental após a radioterapia. A diminuição da saliva é um fator importante na manifestação e desenvolvimento das cáries de radiação, pois a capacidade tampão está severamente limitada, motivando a desmineralização dos tecidos dentais (KROETZ; CZLUSNIAK, 2003; ROLIM et al., 2011).

A saliva é de extrema importância para a manutenção dos tecidos orais, desta maneira, pacientes com um caimento do fluxo salivar, decorrente da radioterapia, tendem a desenvolver, dentre outras alterações uma alta atividade de cárie. A cárie por radiação contorna todo o dente chegando às vezes a atingir os tecidos mais profundos, iniciando de três a quatro meses após a radioterapia e podem apresentar total destruição dos dentes, em até dois anos (SANTOS et al., 2002).

\section{Candidose bucal}

A candidose bucal é uma infecção bem comum nos pacientes em tratamento de neoplasias malignas das vias aero-digestivas superiores, seu agente etiológico é a Candida albicans. Sua manifestação se caracteriza pela presença de placas brancas removíveis a raspagem, que podem apresentar-se na forma pseudomembranosa ou eritematosa. Mesmo sendo um componente da flora bucal, alguns fatores favorecem o aumento desse patógeno, e acaba provocando infecção na mucosa oral. Ocorrerá uma queda no fluxo salivar quando paciente for irradiado, e assim, justifica-se o aumento na ocorrência da candidose, frequentemente também é associada a alterações no paladar e mucosite (JHAM; FREIRE, 2006; FREITAS et al., 2011).

Após as duas primeiras semanas de tratamento, há uma predominação de microorganismos cariogênicos, que ocorre certamente devido à perda da capacidade de limpeza da saliva, do seu efeito tampão, antibacteriano e lubrificante. O comprometimento imunológico desses pacientes facilita também o desenvolvimento de alguns microorganismos. As infecções fúngicas podem permanecer por muitos meses após o tratamento, em especial, nos pacientes que utilizam próteses totais. São colhidas amostras da mucosa labial ou jugal de pacientes antes e durante o tratamento radioterápico, onde se observa a máxima positividade para fungos no decorrer da radioterapia. Essa infecção manifesta-se frequentemente na língua, na mucosa e comissuras labiais, e sua forma mais comum é a pseudomembranosa (RAGGHIANTI et al., 2002).

REFACER v 7. , n. 1, 2018. ISSN - 2317-1367 


\section{Mucosite}

A mucosite é uma das complicações mais frequentes nesses pacientes, os primeiros sintomas observados, surgem na terceira ou quarta semana, caracterizando-se pela inflamação da mucosa oral, irritação nas gengivas, na garganta e até mesmo feridas na boca, edema, eritema, ulceração, presença de pseudomembranas, além de dor e dificuldade na deglutição (LÔBO; MARTINS, 2009). Essa dificuldade em se alimentar pode levar à perda de peso, anorexia, caquexia e desidratação. A depressão e os distúrbios do sono em pacientes são atribuídos devido à mucosite. A mucosite induzida pela radiação aparece em $100 \%$ dos pacientes que recebem radioterapia de cabeça e pescoço (FREITAS et al., 2011).

A Organização Mundial da Saúde definiu uma escala para verificar a mucosite, em que o grau 0 representa a inexistência da mucosite, grau I apresenta se dolorida e eritematosa, grau II apresenta edema, eritema e úlcera dolorosa, e o paciente consegue ainda se alimentar de sólidos, no grau III representa um quadro grave com ulcerações bucais e o paciente se alimenta apenas de líquidos, e o grau IV apresenta uma ulceração profunda, o paciente não consegue se alimentar pela boca, precisando de um suporte nutritivo enteral ou parenteral. A dor é extrema a cada investida em comer ou beber (JHAM; FREIRE, 2006).

\section{Trismo}

O trismo ocorre devido aos músculos mastigatórios limitarem a abertura de boca, conseguindo desenvolver-se no decorrer ou após a radioterapia, se esses músculos permanecerem envolvidos nos campos de tratamento, por causa da fibrose muscular que ocorre em resposta. Esses pacientes que os músculos mastigatórios estão incluídos na irradiação precisam ser orientados a exercitá-los, e abrirem a boca tão alargada possível, umas 20 vezes pelo menos, três vezes por dia. O intuito é para evitar excessiva fibrose muscular e ausência do espaço intersticial (CACCELLI; RAPOPORT, 2008).

O trismo radio-induzido, que se estabelece de 3 a 6 meses após o término do tratamento, sofre um impacto significante na qualidade de vida dos pacientes, sendo um efeito colateral significativo da radioterapia, pois a abertura da boca fica limitada por um período longo, impedindo uma higiene bucal correta. A prevenção do trismo, em vez de seu tratamento, é o objetivo mais desejável (VISSINK et al., 2003; SALAZAR et al.,2008).

\section{Disgeusia}


A disgeusia acontece antes dos sintomas da mucosite, acometendo os pacientes a partir da segunda ou terceira semana de radioterapia, persistindo por várias semanas ou até meses. Ela ocorre devido ao atrofiamento gradual das papilas gustativas, aumentando a viscosidade do fluxo salivar formando uma barreira mecânica de saliva, dificultando o contato entre a língua e os alimentos. Com isso, são perceptíveis os sabores ácidos e amargos, sendo frequentemente afetada no começo da irradiação, em seguida, há alteração gustativa para o sabor doce e salgado (JHAM; FREIRE, 2009; ROLIM et al., 2011).

Os pacientes tentam compensar essas alterações comendo alimentos ricos em açúcar, o que acaba contribuindo com problema de cárie de irradiação, ou usando maior quantidade de temperos, o que aumentaria o incomodo da mucosite. Cerca de $70 \%$ dos pacientes que são sujeitos à radioterapia, resultam também em ausência de apetite e perda de peso, tornando-se a complicação mais incômoda em muitos dos pacientes irradiados. A recuperação geralmente ocorre de 60 a 120 dias após a finalização da irradiação, sendo que o grau da recuperação irá depender da dose recebida de radiação. Essas alterações da sensação são transitórias e reversíveis, havendo retorno da percepção por volta de dois a quatro meses após a radioterapia (CACCELLI; RAPOPORT, 2008; SILVA et al., 2011).

\section{IMPORTÂNCIA DA ATUAÇÃO DO ODONTÓLOGO NA RADIOTERAPIA}

Durante a radioterapia, direciona-se a irradiação para a área do tumor, protegendo as áreas adjacentes, devendo realizar tratamentos preventivos, visando minimizar intercorrências posteriores. A atuação do odontólogo é essencial em todas as fases da radioterapia, tanto nas fases iniciais do diagnóstico, quanto durante o tratamento, realizando avaliações e proporcionando aos pacientes, condições melhores de serem submetidos às modalidades terapêuticas obtendo maiores taxas de cura, dessa forma, reduzindo ou prevenindo os efeitos colaterais (CZLUSNIAK; KROETZ, 2004; CARDOSO et al., 2005).

Os pacientes submetidos à radioterapia para o tratamento do câncer na região de cabeça e pescoço desenvolvem complicações orais. Por esse motivo, assim que a radioterapia for indicada, o paciente deverá ser submetido a uma avaliação da cavidade bucal por um odontólogo (PELISSER et al., 2008). Dessa maneira, é de extrema importância que o odontólogo conheça as diferentes maneiras de tratamento do câncer e as alterações resultantes dessa terapia, para poder preveni-las ou tratá-las, estabelecendo um plano de tratamento adequado para cada paciente (RAGGHIANTI et al., 2002). 
De acordo com Araújo et al. (2009) há estudos que comprovem que em algumas situações o odontólogo não participa ativamente do tratamento do paciente com a doença, sendo por falta de acesso ao profissional, ou por falta de instrução ou demais motivos. É evidente que o campo de atuação do odontólogo é a cavidade bucal, com isso deve ter conhecimento geral do estado de saúde do paciente, que danos o câncer tem proporcionado.

\section{EFEITO DA RADIOTERAPIA SOBRE A QUALIDADE DE VIDA DE PACIENTES COM TUMORES DE CABEÇA E PESCOÇO}

O diagnóstico de câncer, comumente desencadeia reações devastadoras, principalmente de cunho emocional, acarretando sentimentos conflituosos e desequilíbrio, além de gerar um sofrimento intenso capaz de ocasionar em desorganização psíquica, dependendo da localização, do estágio da doença e do tratamento. Mesmo com o avanço da medicina, o diagnóstico é encarado ainda, como sentença de morte. Alguns aspectos da doença podem causar sequências de reações e expectativas no paciente e nos seus familiares. Após o choque no inicio do diagnóstico, os pacientes às vezes apresentam respostas emocionais como raiva, depressão e ansiedade (SILVA et al., 2008).

As reações decorrentes do tratamento causam prejuízo na qualidade de vida dos pacientes e também influenciam na condução e adesão ao tratamento. Essas complicações orais também levam a uma relação negativa na aparência física, dificuldade na comunicação, respiração, causando alterações no humor, levando a ansiedade e depressão, comprometendo na saúde deste paciente (PAULA; SAWADA, 2015). Os pacientes com câncer de cabeça e pescoço, além de estarem com uma doença que ameaça suas vidas, têm de lidar com o impacto dessa e de seu tratamento sobre aspectos estéticos e funcionais (ARAÚJO et al., 2009).

A mortalidade e morbidade referentes à doença maligna são elevadas e causam impacto sobre a qualidade de vida e o custo de tratamento dos pacientes. O câncer de cabeça e pescoço pode atingir a saúde geral e mental, o emprego, a aparência, vida social e vida em família (GALBIATTI et al., 2013). De acordo com Sawada et al. (2006), muitos estudos em relação a qualidade de vida em pacientes com câncer realizando a terapia, têm avaliado esses efeitos indesejáveis do tratamento e auxiliado no programação de intervenções, afim de minimizar, tanto o estresse psicológico quanto o físico para terem melhor reabilitação. 


\section{CONCLUSÃO}

A radioterapia é uma das modalidades terapêuticas de grande importância do câncer na região de cabeça e pescoço, sendo um método eficaz que contribui na melhoria da qualidade de vida, podendo provocar algumas complicações bucais e diversos efeitos colaterais sobre o organismo humano. Esses efeitos colaterais podem surgir durante o tratamento ou anos após a sua finalização, necessitando então de um acompanhamento com um profissional para evitar ou amenizar essas alterações. As principais complicações bucais do câncer de cabeça e pescoço são xerostomia, osteoradionecrose, disgeusia, trismo, mucosite e candidose bucal, ocorrem numa significativa frequência entre os pacientes submetidos, o que acaba dificultando o tratamento.

Complicações devido ao tratamento de tumores na região de cabeça e pescoço surgem devido a vários fatores, sendo o alcoolismo e tabagismo os mais relevantes para a ocorrência dessa neoplasia. Manifestações bucais e efeitos colaterais decorrentes da radioterapia agravam a saúde do paciente, sendo fundamental, a atuação de um odontólogo em todas as fases da terapia. Estudos nesse sentido são de grande importância, seja definindo estratégias de tratamento, ou prevenindo e minimizando o desconforto, proporcionando ao paciente melhor qualidade de vida.

\section{REFERÊNCIAS BIBLIOGRÁFICAS}

ALMEIDA, L. H. R. B.; PEREIRA, Y. B. A. S.; OLIVEIRA, T. A. Radioterapia: percepção de mulheres com câncer cérvico-uterino. Revista Brasileira de Enfermagem, Brasília, v. 61, n. 4, p. 482-87, 2008.

ARAÚJO, S. S. C.; PADILHA, D. M. P.; BALDISSEROTTO, J. Avaliação da condição bucal e da qualidade de vida de pacientes com câncer de cabeça e pescoço atendidos em um hospital público de Porto Alegre. Revista Brasileira de Cancerologia, Rio de Janeiro, v. 55, n. 2, p. 129-38, 2009.

ARISAWA, E. A. L.; SILVA, C. M. O. M.; CARDOSO, C. A. C.; LEMOS, N. R. P.; PINTO; M. C. Efeitos colaterais da terapia antitumoral em pacientes submetidos à químio e à radioterapia. Revista Biociências, Taubaté, v. 11, n. 1-2, p. 55-61, jan.-jun. 2005. 
BRAGANTE, K. C.; NASCIMENTO, D. M.; MOTTA, N. W. Avaliação dos efeitos agudos da radioterapia sobre os movimentos mandibulares de pacientes com câncer de cabeça e pescoço. Revista Brasileira de Fisioterapia, Porto Alegre, 2011.

BRENTANI; M. M.; COELHO, F. R. G.; KOWALSKI, L. P. Bases da oncologia. $2^{\text {a }}$ Edição, São Paulo: Lemar Livraria; Editora Marina e Tecmedd, 2003.

CACCELLI, É. M. N.; RAPOPORT; A. Para-efeitos das irradiações nas neoplasias de boca e orofaringe. Revista Brasileira de Cirurgia de Cabeça e Pescoço, São Paulo, v. 37, n. 4, p. 198-201, out.-nov.-dez. 2008.

CARDOSO, M. F. A.; NOVIKOFF, S.; TRESSO, A.; SEGRETO, R. A.; CERVANTES, O. Prevenção e controle das sequelas bucais em pacientes irradiados por tumores de cabeça e pescoço. Radiologia Brasileira, São Paulo, v. 38, n. 2, p. 107-15, 2005.

CASTRO, G. Câncer de cabeça e pescoço é um dos tumores mais frequentes em homens. 2017.

COLOMBO, J.; RAHAL, P. Alterações genéticas em câncer de cabeça e pescoço. Revista Brasileira de Cancerologia, Rio de Janeiro, v. 55, n. 2, p. 165-174, 2009.

CZLUSNIAK, G. D.; KROETZ, F. M. Alterações bucais e condutas terapêuticas em pacientes infanto-juvenis submetidos a tratamentos anti-neoplásicos. Publicatio UEPG Ciências Biológicas e da Saúde, Ponta Grossa, v. 9, n. 2, p. 41-48, jun. 2003.

FREITAS, D. A.; CABALLERO, A. D.; HERRERA, A. H.; MERCADO, L. F.; FREITAS F. A.; ANTUNES S. L. O. A saúde oral e a radioterapia de cabeça e pescoço. Arquivos Catarinenses de Medicina, Florianópolis, v. 40, n. 3, 2011.

FREITAS, D. A.; CABAllERO, A. D.; PEREIRA, M. M.; OLIVEIRA, S. K. M.; SILVA, G. P.; HERNÁNDEZ, C. I. V. Sequelas bucais da radioterapia de cabeça e pescoço. Revista CEFAC, Minas gerais, v. 13, n. 6, p. 1103-08, nov.-dez. 2011.

FRIGATO, S.; HOGA, L. A. K. Assistência à mulher com câncer de colo uterino: o papel da enfermagem. Revista Brasileira de Cancerologia, São Paulo, v. 49, n. 4, p. 209-14, 2003.

GALBIATTI, A. L. S.; PADOVANI-JUNIOR, J. A.; MANÍGLIA, J. V.; RODRIGUES, C. D. S.; PAVARINO, É. C.; GOLONI-BERTOLLO, E. M. Head and neck cancer: causes, 
prevention and treatment. Brazilian Journal of Otorhinolaryngology, São Paulo, v. 79, n. 2, p. 239-47, 2013.

GRIMALDI, N.; SARMENTO, V.; PROVEDEL, L.; ALMEIDA, D.; CUNHA, S. Conduta do cirurgião-dentista na prevenção e tratamento da osteorradionecrose: revisão de literatura. Revista Brasileira de Cancerologia, Rio de Janeiro, v. 51, n. 4, p. 319-324, ago. 2005.

INCA- INSTITUTO NACIONAL DE CÂNCER JOSÉ ALENCAR GOMES DA SILVA. Doenças Relacionadas ao Tabagismo. 2017.

INCA- INSTITUTO NACIONAL DE CÂNCER JOSÉ ALENCAR GOMES DA SILVA. Estimativa 2016 Incidência de Câncer no Brasil.

INCA - INSTITUTO NACIONAL DE CÂNCER JOSÉ ALENCAR GOMES DA SILVA. ABC do Câncer Abordagens Básicas Para Controle Do Câncer. 2011.

JHAM, B. C.; FREIRE, A. R. S. Complicações bucais da radioterapia em cabeça e pescoço. Revista Brasileira de Otorrinolaringologia, São Paulo, v. 5, n. 72, p. 704-08, 2006.

KARKOW, M. C.; GIRARDON-PERLINI, N. M. O.; MISTURA , C.; ROSA, B. V. C.; ROSA, N.; MARTINS, M. S.; DEBUS, DORNELES, P. S.; V. C. Perfil dos usuários do serviço de radioterapia de um hospital universitário. Revista de Enfermagem Universidade Federal de Santa Maria, Rio Grande do Sul, v. 3, Esp. p. 636-646, 2013.

LÔBO, A. L. G.; MARTINS, G. B. Consequências da Radioterapia na Região de Cabeça e Pescoço: Uma Revisão da Literatura. Revista Portuguesa de Estomatologia, Medicina Dentária e Cirurgia Maxilofacial, Salvador, v. 50, n. 4, p. 251-55, 2009.

MARTA, G. N.; HANNA, S. A.; SILVA, J. L. F.; CARVALHO, H. A. Câncer de cabeça e pescoço e radioterapia: breve contextualização. Diagn Tratamento, São Paulo, v. 16, n. 3, p. 134-6, 2011.

MONTEIRO, L.; BARREIRA, E.; MEDEIROS, L. Osteorradionecrose dos Maxilares. Revista Portuguesa de Estomatologia, Medicina Dentária e Cirurgia Maxilofacial, São Paulo, v. 46, n. 1, p. 49-62 2005.

PAULA, J. M,; SAWADA, N. O. Qualidade de vida relacionada à saúde de pacientes com câncer em tratamento radioterápico. Revista Rene, São Paulo, v.16, n.1, p. 106-113, jan-fev. 2015. 
PELISSER, F., ROCKENBACH, M. I., FIGUEIREDO, M. A., CHERUBINI, K., \& VEECK, E. B. Considerações sobre as modalidades radioterapêuticas empregadas para o tratamento de neoplasias na região de cabeça e pescoço. Revista da Faculdade de Odontologia, Passo Fundo, v.13, n. 1, p. 75-79, jan.-abr. 2008.

RAGGHIANTI, M. S.; GREGHI, S. L. A.; CAPELOZZA, A. L. ARESENDE, D. R. B. Tratamento radioterápico: Parte I - Reações adversas na cavidade bucal. Salusvita, Bauru, v. 21, n. 1, p. 77-86, 2002.

ROLIM, A. E. H.; COSTA, L. J.; \& RAMALHO, L. M. P. Repercussões da radioterapia na região orofacial e seu tratamento. Radiologia Brasileira, Salvador, v.44, n. 6, p. 388-95, nov.-dez. 2011.

SALAZAR, M.; VICTORINO, F. R.; PARANHOS, L. R.; RICCI, I. D.; GAETI, W. P.; CAÇADOR, N. P. Efeitos e tratamento da radioterapia de cabeça e pescoço de interesse ao cirurgião dentista Revisão da literatura. Revista Odonto, São Bernardo do Campo, v. 16, n. 31, p. 62-68, 2008, jan.-jun. 2008.

SANTOS, A. C. M.; NOGUTI, F.; MENDES, F. C. A.; ALBORGHETTI, G. P.; PEREIRA, M. B. Complicações Bucais no Tratamento Radioterápico. 2002.

SANTOS, M. G.; SILVA, L. C. F.; LINS, C. A.; PASSOS, D. D.; OLIVEIRA NETO, J. N.; SANTOS, T. S. Fatores de risco em radioterapia de cabeça e pescoço. Revista Gaúcha de Odontologia, Porto Alegre, v. 58, n. 2, p. 191-96, abr.-jun. 2010.

SAWADA, N. O.; DIAS, A. M.; ZAGO, M. M.F. O efeito da radioterapia sobre a qualidade de vida dos pacientes com câncer de cabeça e pescoço. Revista Brasileira de Cancerologia, Ribeirão Preto, v. 52, n. 4, p. 323-29, 2006.

SASSI, L. M.; MACHADO, R. A. Protocolo pré-radioterapia de cabeça e pescoço. Revista Brasileira de Cirurgia de Cabeça e Pescoço, São Paulo, v. 38, nº 3, p. 208-210, jul.-ago.set. 2009.

SILVA A. I. V.; GALANTE C., MANZI F. R. Efeito da radiação ionizante sobre o paladar em pacientes submetidos à radioterapia para a região da cabeça e pescoço. Radiologia Brasileira, São Paulo, v. 44, n. 5, p. 297-300, set.-out. 2011.

SILVA, S. S.; AQUINO, T. A. A.; SANTOS, R. M. O paciente com câncer: cognições e emoções a partir do diagnóstico. Revista Brasileira De Terapias Cognitivas, Ribeirão Preto, v. 4, n. 2, 2008. 
VIDAL, A. K. L.; REVOREDO, E. C. V. Radioterapia em tumores na boca. Revista Odontologia Clínico-Científica, Recife. v. 9, n. 4, p. 295-298, out.-dez. 2010.

VISSINK, A.; BURLAGE, F.R.; SPIJKERVET, F.K.L.; JANSMA J.; COPPES, R.P. prevention and treatment of the consequences of head and neck radiotherapy. Critical Reviews in Oral Biology \& Medicine, v. 14, n. 3, p. 213-25, 2003. 